\title{
ULTRA-HIGH STRAIN RATE CONSTITUTIVE MODELING OF PURE TITANIUM USING PARTICLE IMPACT TEST
}

\author{
A Thesis \\ Presented to the Faculty of the Graduate School \\ of Cornell University \\ In Partial Fulfillment of the Requirements for the Degree of \\ Master of Science in Mechanical Engineering
}

by

Xuchen Wang

May2020 
(C) 2020 Xuchen Wang 


\begin{abstract}
With the advent of advanced testing strategies like laser-induced particle impact test, it is possible to study materials mechanics under extremely high deformation rates, i.e., above $10^{6} \mathrm{~s}^{-1}$, a relatively less explored regime of strain rates. In this study, we accelerate microparticles of commercially pure titanium to $\sim 100 \mathrm{~m} / \mathrm{s}$ towards a rigid substrate and record their deformation upon impact in real time. We also conduct finite element modeling of the experimentally recorded impacts using two constitutive equations: Johnson-Cook and Zerilli-Armstrong. We show that titanium microparticles experience strain rates in the range of $10^{6}-10^{10} \mathrm{~s}^{-1}$ upon impact. We evaluate the capability of the Johnson-Cook and Zerilli-Armstrong equations in predicting material response at ultra-high strain rates. With an optimization-based constitutive modeling approach, we also propose updated strain rate-related constitutive parameters for both equations that can improve the extent to which they can successfully describe the deformation of materials at higher strain rates.
\end{abstract}




\section{BIOGRAPHICAL SKETCH}

Xuchen Wang was born and raised in Zhejiang, China, where he finished his primary and secondary education. He obtained a Bachelor of Science Degree in Aerospace Engineering at University of California, San Diego in 2018, after which he pursued a Master of Science Degree in Mechanical Engineering with a research focus on

material science and solid mechanics at Cornell University, graduating in May 2020. 
爸爸妈妈, 谢谢你们! 我爱你们! 


\section{ACKNOWLEDGMENTS}

I acknowledge the continued guidance and support from Dr. Mostafa Hassani, my supervisor, over the past two years. Dr. Mostafa Hassani and I also acknowledge a cooperative research agreement with the US Army Research Laboratory, Contract: W911NF-15-2-0034, "Development of Additive Manufacturing and Advanced Materials Processing for the DOD”. 


\section{TABLE OF CONTENTS}

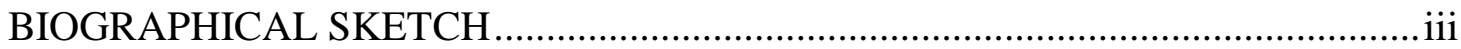

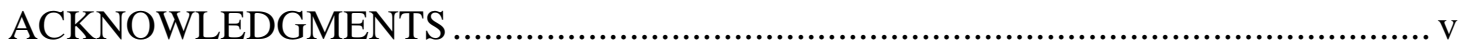

TABLE OF CONTENTS ............................................................................... vi

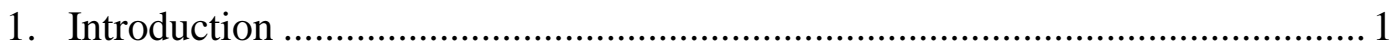

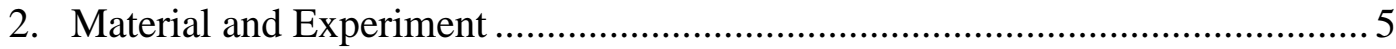

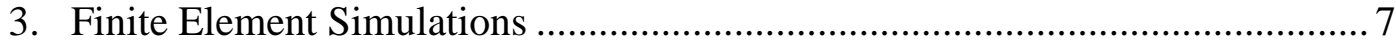

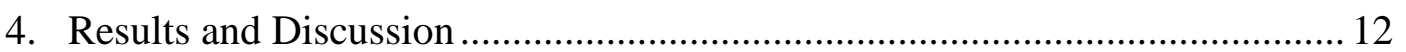

4.1 Real-time Observations of Deformation.............................................. 12

4.2 Finite Element Results ...................................................................... 13

4.3 Calibration of Constitutive Parameters.................................................... 14

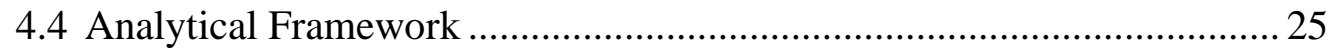

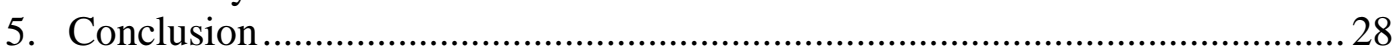

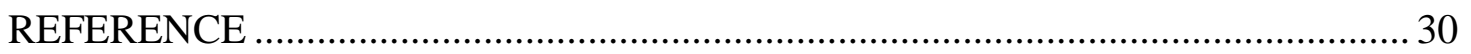




\section{Introduction}

Since plastic deformation of metals and alloys shows significant strain rate dependence, it is imperative to establish accurate material models and parameters to model and predict material response, especially under dynamic loading conditions. Examples of such deformation include ballistic impacts, impulsive loadings, space dust particle impacts, as well as manufacturing processes such as machining, shot peening, and cold spray coating [1-6]. Strain rates involved in these examples are generally higher than $10^{3} \mathrm{~s}^{-1}$, and in some cases even as high as $10^{9} \mathrm{~s}^{-1}$, i.e., orders of magnitudes higher than the strain rate regimes commonly probed in standard mechanical testing procedures [7].

To study the strain-rate sensitivity of flow stress, various experimental techniques have been developed including Taylor Impact Test, Kolsky (Split-Hopkins Pressure) Bar, and plate impact test [8-10]. Figure 1 shows how these high strain rate experiments measure flow stress in different regions of the characteristic size-velocity map compared to the conventional macroscale tensile testing. In this figure, the strain rate appears as a series of contours defined by the ratio of velocity and size. This suite of high strain rate experiments can be used to inform and calibrate a variety of empirical and physical constitutive equations to precisely model the plastic deformation under more complex states of stress. 


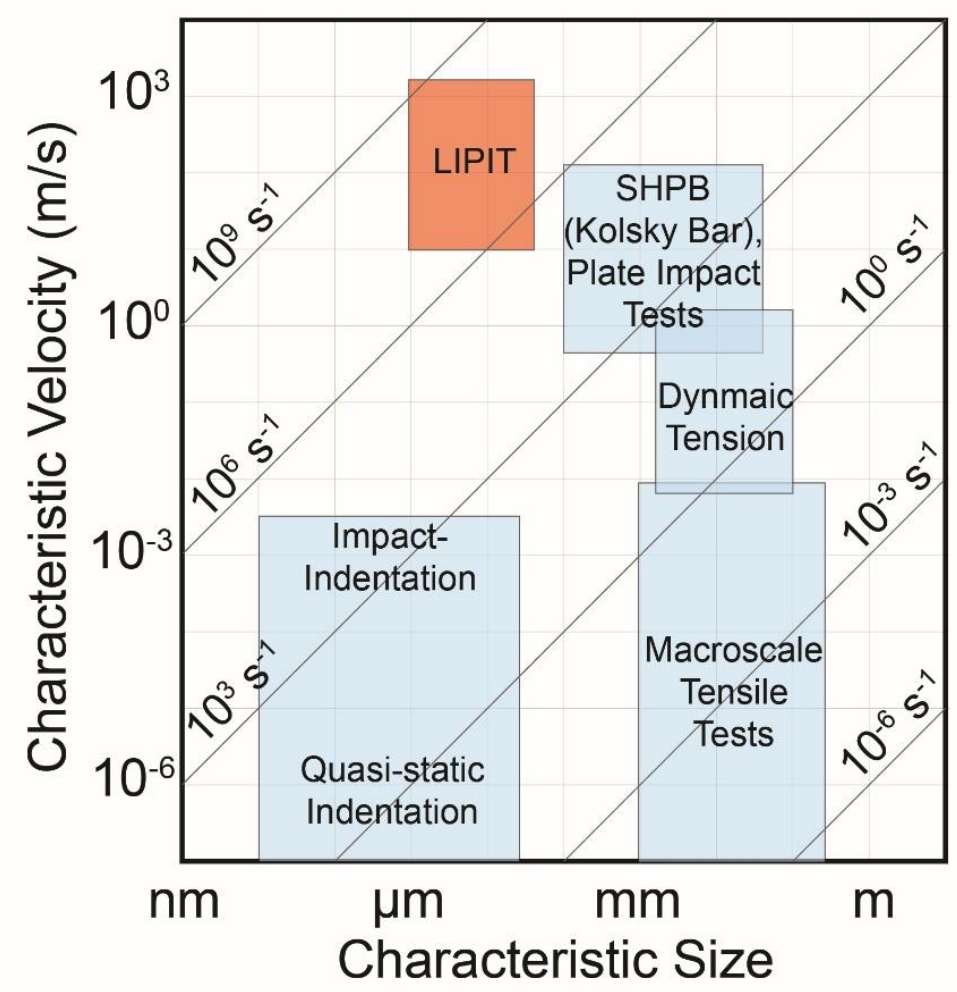

Figure 1: Characteristic size-velocity map showing experimental techniques and the corresponding strain rates.

Starting from lower strain rates, for example, Murugesan et al. [11], and SheikhAhmad et al. [12] performed tension and torsion tests on AISI 1045 medium carbon steel, and commercially pure $(\mathrm{CP})$ titanium respectively to calibrate the Johnson-Cook plasticity model. They found that the Johnson-Cook plasticity model can successfully describe the plastic deformation in the $10^{-2} \mathrm{~s}^{-1}-10^{2} \mathrm{~s}^{-1}$ strain rate regime. Strain rates at $10^{3} \mathrm{~s}^{-1}-10^{4} \mathrm{~s}^{-1}$ are typically induced in the Kolsky (Split-Hopkins Pressure) Bars test, such as the works done by Simon et al., Chichili et al., and Mishra et al. [13-15]. Simon et al. conducted both quasi-static and dynamic loading experiments at various strain rates and temperatures to calibrate material parameters for three constitutive 
relations: Johnson-Cook (JC), Voyiadjis-Abed (VA), and Rusinek-Klepaczko (RK) [13]. They observed that the empirical JC model starts to fail at extreme strain rates and temperatures and that the two semi-physically based models (VA and RK) improve the simulation response in terms of thermal sensitivity. Chichili et al. have shown that the $\alpha$-titanium exhibits significant strain rate sensitivity, especially at high strain rates [14]. Similar behavior in copper is observed by Mishra et al [15]. Gangireddy [16] also used Kolsky Bar test on $\mathrm{CP}$ Ti and proposed a modified Johnson-Cook plasticity model to account for the allotropic phase transformation of $\mathrm{CP}$ Ti at high temperatures $\left(>880{ }^{\circ} \mathrm{C}\right)$. Meyer, Jr. [17] conducted Kolsky Bar experiments to investigate the localization behavior of Ti-6Al-4V using a modified Zerilli-Armstrong plasticity model to account for the dominant hexagonal close packed (HCP) crystal structure in Ti-6Al-4V as well as to successfully address shear instability in material response. Taylor Impact test, done by Hernandez et al. [18] can reach strain rates as high as $10^{6} \mathrm{~s}^{-1}$ to $10^{7} \mathrm{~s}^{-1}$, where they calibrated material models for 1018 steel for the Cowper-Symonds material model.

In the above examples, the material is subjected to one dominant stress component. The second suite of experimental techniques to characterize material behavior at high strain rates is based on dynamic impressions and indentations where a 3D state of stress drives the deformation. For example, Dean et al. [19] and Burley et al. [20] developed an approach to obtain material parameters for copper that can reproduce high-quality simulation results through iterative finite element (FE) modeling by spherical indentation and ballistic impact experiments that can reach strain rates of $10^{4}$ 
$\mathrm{s}^{-1}$ to $10^{6} \mathrm{~s}^{-1}$. Andrews et al. [21] investigated material response using data from impact experiments of a sharp indenter at strain rates of $\sim 10^{5} \mathrm{~s}^{-1}$ and showed that strain rate sensitivity increases in several materials starting at $10^{3} \mathrm{~s}^{-1}$. A similar conclusion is found in the work by $\mathrm{Lu}$ et al. [22], where they verified the strain rate sensitivity of oxygen-free high-conductivity (OFHC) copper using dynamic indentation at strain rates of $10^{2} \mathrm{~s}^{-1}$ to $10^{3} \mathrm{~s}^{-1}$. Other material properties such as dynamic hardness can also be studied using this type experimental method. For instance, Trelewicz et al. [23] showed that at strain rate of $10^{3} \mathrm{~s}^{-1}$, the Hall-Petch relationship, one that relates a material's strength to its grain size, breaks down at a grain size below 10 to $20 \mathrm{~nm}$. While the above methods have provided invaluable insight into material responses at high deformation rates, they remain largely limited to strain rate regimes up to $10^{6} \mathrm{~s}^{-1}$ leaving constitutive parameters of materials largely quantitatively unexplored at higher strain rates.

Furthermore, while a good number of works used the aforementioned techniques to investigate the behavior of pure titanium at high strain rates [12,14,24], titanium constitutive parameters are rarely calibrated for the ultra-high strain rates $\left(10^{6}-10^{9} \mathrm{~s}^{-1}\right)$. This regime of strain rate is particularly relevant to what is induced in the material during cold spray processing $[1,3,25]$. Cold spray is a coating deposition process in which metallic particles are accelerated to $10^{2}$ to $10^{3} \mathrm{~m} / \mathrm{s}$ to impact onto metallic substrates. The particles undergo extreme plastic deformation and can adhere to the surface upon impact, balding up coatings or bulk materials. The applications of cold spray have extended from surface engineering to surface repair and to additive manufacturing in recent years with an increasing interest in deposition of pure 
titanium and its alloys. Accurate knowledge of plastic deformation at high strain rates is needed in order to predict the behavior of titanium powder particles upon impact and to develop optimum deposition windows.

High strain rates beyond $10^{6} \mathrm{~s}^{-1}$ can be induced in materials by impact velocities on the order of $\sim 100 \mathrm{~m} / \mathrm{s}$ while decreasing the characteristic size of the material to micronscales $[25,26]$. Inspired by the classical Taylor impact test, we employ laser-induced projectile impact test (LIPIT) $[25,27-30]$ to accelerate micron-scale spherical particles of titanium and study their deformation upon impact to rigid substrates in real-time. As indicated in Fig. 1 the combination of impact velocities and length scales are such that we can effectively achieve strain rates in the range of $10^{6}-10^{9} \mathrm{~s}^{-1}$. We conduct finite element simulations of impacts combined with an optimization scheme to calibrate the strain rate-related material parameters that can reproduce the experimentally observed deformations for two constitutive models, namely, JohnsonCook and Zerilli-Armstrong.

\section{Material and Experiment}

The impact experiments were conducted using an all-optical microballistic platform [25,27-30], shown schematically in Fig. 2A. The impacting titanium particles are dispersed on top of a launch pad consisting of a $30-\mu \mathrm{m}$ thick polyurea film, under which a 60 -nm thick intermediate gold layer and a $210-\mu \mathrm{m}$ thick glass support exist. The intermediate gold layer is ablated by a 532-nm wavelength laser pulse with a 10ns duration and a $50-\mu \mathrm{m}$ spot size. The ablation of the gold film drives the top polyurea layer forward and in turn accelerates a titanium particle toward an aluminum 
oxide target acting as a rigid substrate. The impact velocity at which the particle travels can be changed by adjusting the laser energy (from 2 to $60 \mathrm{~mJ}$ ). The distance between the launching pad and the substrate is typically $1 \mathrm{~mm}$. We capture the particle approach, impact on the substrate, and its rebound in real-time using a $\mu$ s laser pulse (10- $\mu$ s duration, 640-nm wavelength) for illumination and a high-speed camera (SIMX 16, Specialised Imaging).
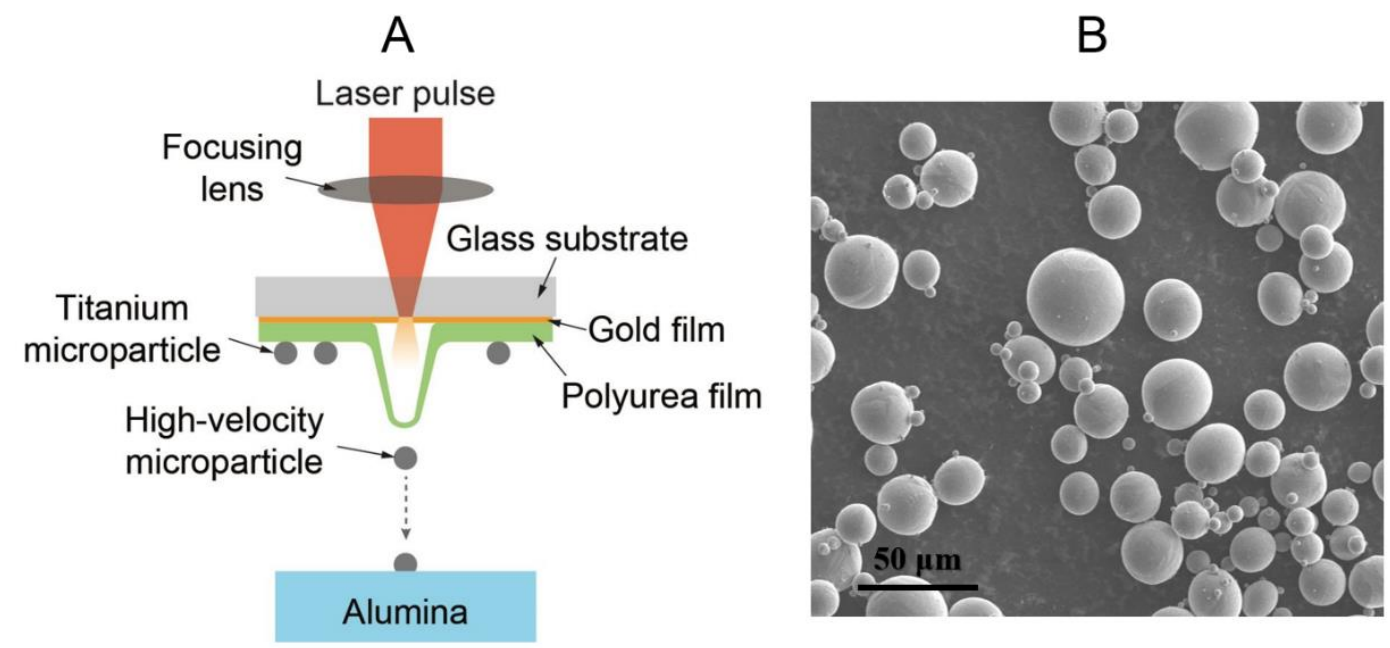

Figure 2: (A) Experimental setup that has a launchpad consisting of three layers on which impact particles are dispersed and accelerated towards a substrate, and (B) scanning electron microscopy (SEM) image of titanium powder particles.

An alumina oxide plate $(10 \times 10 \times 1 \mathrm{~mm})$ was purchased from Alfa Aesar (Ward Hill, USA) and used as a rigid flat substrate in our impact experiment. The surface roughness of the alumina oxide plate, measured by atomic force microscopy, is less than $0.5 \mathrm{~nm}$. A batch of plasma-atomized spherical Ti powder particles with a nominal size of -325 mesh $(<44 \mu \mathrm{m})$ was purchased from AP\&C (Quebec, Canada). The 
scanning electron microscopy observation of the powder particles, shown in Fig. 2B, demonstrates a spherical morphology and a smooth surface for the particles. The diameter of the selected particle from the batch was measured to be $48 \mu \mathrm{m}$ prior to launch using a secondary CCD camera before the launch.

\section{Finite Element Simulations}

A 2D axisymmetric coupled thermomechanical dynamic explicit FE model that consists of a deformable impacting particle and a rigid non-deformable substrate was created using Abaqus 2018 [31]. Figure 3 shows a general geometry of the model, which includes a half-circle representing the impacting particle and a rigid line representing the substrate. A range of particle sizes, 22.4-67.2 $\mu \mathrm{m}$, was considered to replicate the experimental conditions. While the substrate is meshed with a 2-node linear axisymmetric rigid link element (RAX2), the impacting particle is structurally meshed using 4-node thermally coupled axisymmetric quadrilateral (CAX4RT) elements with an element size of $1.5 \mu \mathrm{m}$ set after trial mesh sensitivity analysis. While in reality, nanoscale asperities could be present on both particle and substrate surfaces, we have assumed their effect is negligible[32] on the continuum response of the microparticle in view of them being several orders of magnitude smaller than the length scale of the problem at hand.

The substrate was fixed in all degrees of freedom, and symmetry boundary condition was applied to the axis of symmetry of the impacting particle. The initial temperature of the particle was set to $296 \mathrm{~K}$. A range of initial velocities, $144-428 \mathrm{~m} / \mathrm{s}$, measured experimentally, was applied to the particle in the negative y-direction to replicate the 
experimental conditions. The gravitational acceleration is ignored due to the high impact velocity, on the order of $\sim 10^{2} \mathrm{~m} / \mathrm{s}$, and the contact between the particle and the substrate is considered frictionless. Plastic work was assumed to be dissipated as heat with a conversion factor of $90 \%$ while no heat transfer was considered to occur between the particle and the substrate. In our simulations, the total energy is conserved. The kinetic energy of the incoming particle is partially converted to plastic work and the remaining is responsible for the rebound of the particle. A total time of 100 ns was set for the model within which the particle was allowed enough time to impact the substrate and fully rebound.

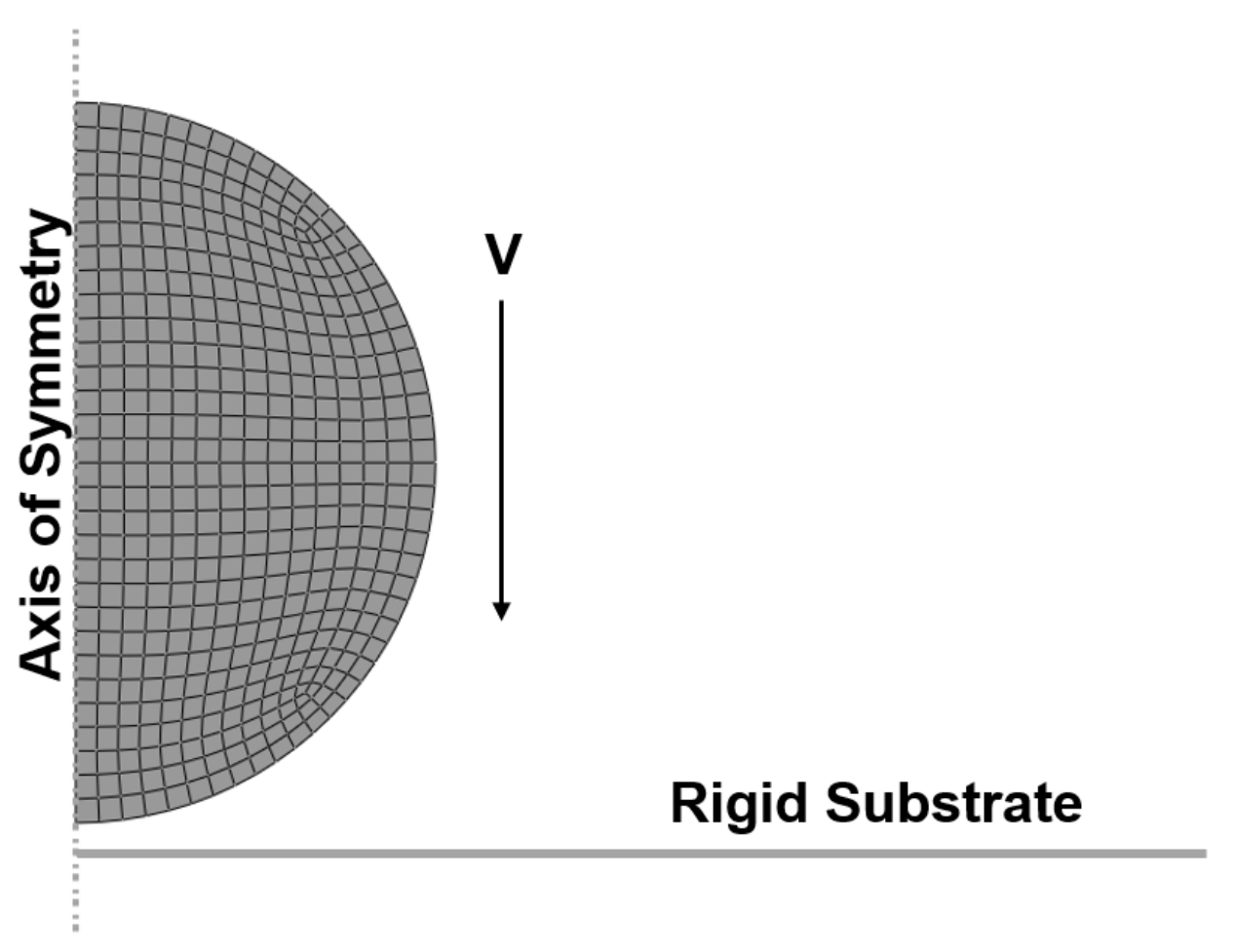

Figure 3: Schematics of the FEA model for the impact experiment.

The density, Young's Modulus, and Poisson's ratio of the titanium particle were set to $4510 \mathrm{~kg} / \mathrm{m}^{3}, 116 \mathrm{GPa}$, and 0.34 , respectively. The thermal properties including 
conductivity and specific heat are assumed to be constant and set to $17 \mathrm{~W} \cdot \mathrm{m}^{-1} \cdot \mathrm{K}^{-1}$ and $528 \mathrm{~J} \cdot \mathrm{kg}^{-1}$ respectively. We have considered the substrate to be a rigid material to reduce the computational cost associated with a large number of simulations for optimization that will be discussed later. We have justified our assumption by conducting trial finite element simulations of impact with a rigid substrate and comparing the results with the case where we changed the substrate to be elastic (with $370 \mathrm{GPa}$ Young's modulus and 0.22 Poisson's ratio). The difference between the rebound velocities was found to be $\sim 0.5 \%$. We have also observed a very close match between deformed particle geometries in the two cases. We, therefore, proceed confident in that the assumption of a rigid substrate does not have a significant effect on the results.

For the plastic regime, we implement constitutive equations that are capable of predicting the material response under high deformation rate. Two of the most widely used material models are the Johnson-Cook (JC) [33] and the Zerilli-Armstrong (ZA) [34] plasticity models. Both models are established to describe plastic deformation of materials under high strain rate loading, and to incorporate dependence on the plastic strain, temperature, and most importantly in our work, strain rate. However, while the JC model is purely empirical, the ZA model is mechanistically based. Despite being purely empirical, the JC model has proven successful in predicting material behavior at strain rates up to $10^{3}-10^{4} \mathrm{~s}^{-1}[35,36]$. The ZA model, on the other hand, has successfully reproduced material deformation at higher strain rates [37,38]. 
The JC model requires 5 parameters, which is usually determined by curve fittings to experimental measurements. Equation 1 shows the dependence of the flow stress on the plastic strain, strain rate, and temperature in the JC plasticity model, which utilizes J2 plasticity:

$$
\sigma_{y}\left(\varepsilon_{p}, \dot{\varepsilon_{p}}, T\right)=\left[A+B\left(\varepsilon_{p}\right)^{n}\right]\left[1+C \ln \left({\dot{\varepsilon_{p}}}^{*}\right)\right]\left[1-\left(T^{*}\right)^{m}\right]
$$

where $\varepsilon_{p}$ is the equivalent plastic strain, $\dot{\varepsilon_{p}}$ the plastic strain rate, $\mathrm{T}$ is the temperature, and $\mathrm{A}, \mathrm{B}, \mathrm{C}, \mathrm{n}, \mathrm{m}$ are material constants. Also, ${\dot{\varepsilon_{p}}}^{*}$ and $T^{*}$ are two dimensionless parameters defined as:

$$
\dot{\varepsilon}_{p}^{*}=\frac{\dot{\varepsilon_{p}}}{\dot{\varepsilon_{p 0}}} \quad \text { and } \quad T^{*}=\frac{\left(T-T_{0}\right)}{\left(T_{m}-T_{0}\right)}
$$

where $\dot{\varepsilon}_{p 0}^{\cdot}$ is the reference strain rate at which the quasi-static parameters $(\mathrm{A}, \mathrm{B}$ and $\mathrm{n})$ are determined and is usually set to $1 \mathrm{~s}^{-1} . \mathrm{T}_{0}$ is the reference temperature, and $\mathrm{T}_{\mathrm{m}}$ is the melting temperature of the material.

The ZA model, on the other hand, is based on the thermally activated dislocation motion of material undergoing plastic deformation. Zerilli and Armstrong used the notion that the activation volume of face-centered cubic (FCC) metal is dependent on strain while that of body-centered cubic (BCC) is independent of strain. As a result, the model takes different functional forms for FCC and BCC materials. They first proposed constitutive equations for FCC and BCC crystal structures [34], and later established that HCP metals exhibit deformation responses that are in between the other two types of materials [24,35,39]. Therefore, in the case of titanium with HCP 
crystal structure, the ZA model is a superposition of the FCC and BCC constitutive equations as shown in Eq. 3:

$$
\begin{aligned}
\sigma_{y}=C_{0}+B_{0} \varepsilon^{C_{n}} \exp \left[-\alpha_{0} T+a_{1} T \ln (\dot{\varepsilon})\right] \\
+B \exp \left[-\beta_{0} T+\beta_{1} T \ln (\dot{\varepsilon})\right](\text { for HCP metals })
\end{aligned}
$$

where $\varepsilon_{p}$ is the equivalent plastic strain, $\dot{\varepsilon}_{p}$ the plastic strain rate, $\mathrm{T}$ is the temperature, and $C_{0}, B_{0}, C_{n}, \alpha_{0}, \alpha_{1}, B, \beta_{0}, \beta_{1}$ are material constants. A VUHARD Abaqus user subroutine [31] was developed to implement the ZA constitutive equation in the FE simulations. The material parameters $[16,24,40]$ used for each model are summarized in Table 1.

Table 1: Material parameters for CP Ti.

\begin{tabular}{cc|cc}
\hline \multicolumn{2}{c|}{ JC Parameters } & \multicolumn{2}{c}{ ZA Parameters } \\
\hline $\mathrm{A}(\mathrm{MPa})$ & 390 & $\mathrm{C}_{0}(\mathrm{MPa})$ & 390 \\
$\mathrm{~B}(\mathrm{MPa})$ & 481.61 & $\mathrm{~B}_{0}(\mathrm{MPa})$ & 990 \\
$\mathrm{n}$ & 0.319 & $\mathrm{C}_{\mathrm{n}}$ & 0.5 \\
$\mathrm{C}$ & 0.0194 & $\mathrm{~B}(\mathrm{MPa})$ & 700 \\
$\mathrm{~m}$ & 0.655 & $\alpha_{0}\left(\mathrm{~K}^{-1}\right)$ & $1.1 \times 10^{-4}$ \\
$\mathrm{~T}_{\mathrm{m}}(\mathrm{K})$ & 1923 & $\alpha_{1}\left(\mathrm{~K}^{-1}\right)$ & $7.5 \times 10^{-5}$ \\
& & $\beta_{0}\left(\mathrm{~K}^{-1}\right)$ & $2.24 \times 10^{-3}$ \\
& & $\beta_{1}\left(\mathrm{~K}^{-1}\right)$ & $9.73 \times 10^{-5}$ \\
\hline
\end{tabular}

By calibrating the parameters that are directly related to strain rate dependency, namely $\mathrm{C}$ for the JC equation and $\alpha_{1}$ and $\beta_{1}$ for the ZA equation, we shall see both the 
effect of strain rate sensitivity imposes on the material response in the high strain rate regime, and the effectiveness of each model to predict such deformation.

\section{Results and Discussion}

\subsection{Real-time Observations of Deformation}

The central idea of the optimization in this study is to search strategically in a range for a single, or a set of material parameters, that produces a deformed particle geometry that best matches the experimentally obtained profile. Figure 4 shows snapshots from the impact experiment used to resolve the deformed particle geometry. The figure illustrates a time span of 450 ns during which a titanium particle, measured $48 \pm 1 \mu \mathrm{m}$ in diameter and $190 \pm 5 \mathrm{~m} / \mathrm{s}$ in impact velocity, impacts a rigid alumina substrate and rebounds after plastic deformation. Here we can see that the duration of contact between the spherical particle and the rigid substrate is very short (less than 100ns). As a result of the impact, the initially spherical particle transforms into a symmetric dome shape, from which we can clearly see that the deformation is largely localized at the bottom half of the particle. Therefore, the upper half of the particle undergoes very little, if any, deformation, making it relatively easy for the measurement and reconstruction of the experimental results; the particle's geometry after it fully rebounds can be reconstructed and set as the reference deformed geometry for optimization. 


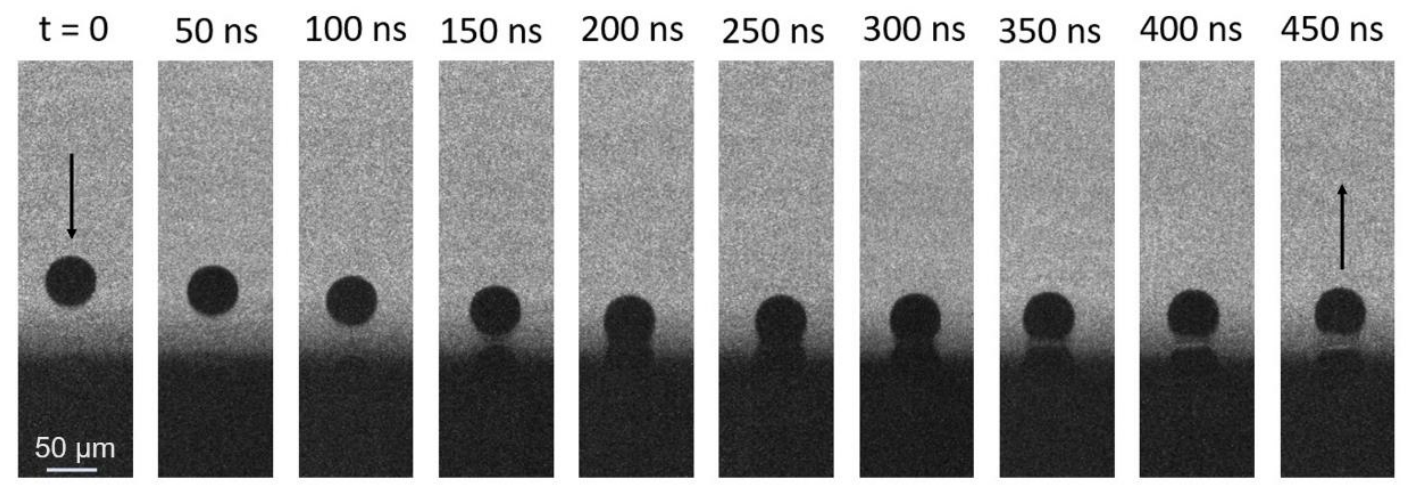

Figure 4: Snapshots of an impact experiment showing a titanium particle impacting a rigid substrate and rebounding after plastic deformation.

\subsection{Finite Element Results}

Figure 5 illustrates impact-induced deformation from FEA simulations using the ZA model with material parameters from the literature. From the simulations, we see that the contact time between the two instances is extremely short (less than 100ns). Figure 5A shows localized plasticity developing in the lower half of the particle which agrees with the experimental observations. The heat generated in the particle is mainly accumulated in the same region and does not have enough time to be conducted away during contact as a result of the adiabaticity of this high strain rate deformation. We note that the rise in temperature is not large enough to likely have a significant impact on the thermal and elastic properties of the particle which assumed to be constant. By extracting the nodal coordinates of the deformed geometry and by comparing particle profiles from both the experiment and simulations, we can see how successful the material models are in terms of describing the deformation. 

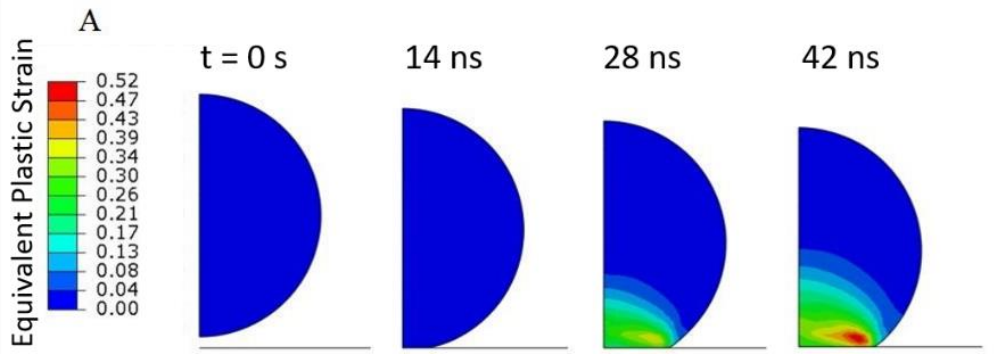

$56 \mathrm{~ns}$

70 ns

B
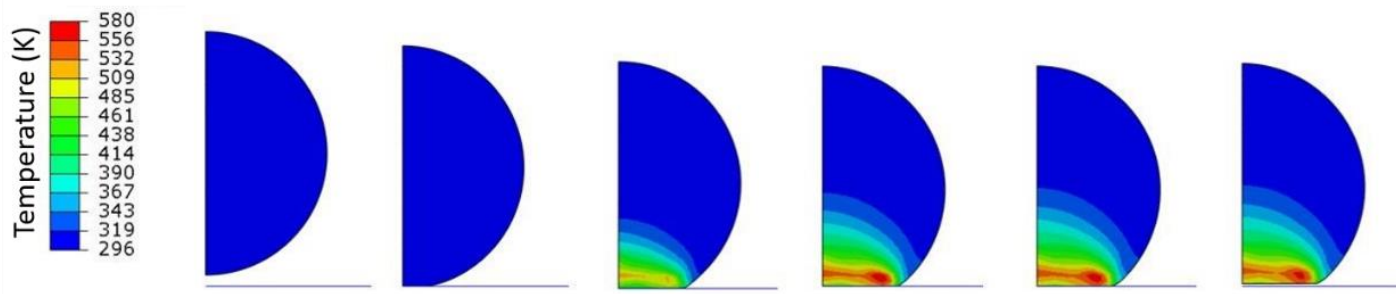

$50 \mu \mathrm{m}$

Figure 5: FEA model of the particle impact experiment using the ZA model with material parameters from the literature showing the evolution of (A) equivalent plastic strain and (B) temperature within the particle during impact-induced deformation.

\subsection{Calibration of Constitutive Parameters}

Figure 6A shows a comparison between the experimentally measured geometry and those obtained by FE modeling using JC and ZA constitutive equations with proposed parameters from the literature. Both the experimental and simulated geometries were reproduced for the fully rebounded particle. The error bars associated with the experimental measurements represent an uncertainty of 4 pixels, in our case resulting in an absolute value of $\pm 1.26 \mu \mathrm{m}$ in both $\mathrm{X}$ and $\mathrm{Y}$ coordinates. From the comparison in Fig. 6A, we note that the ZA model results in a better match with the experiment compared to the JC model. The deformed geometry produced by the JC model using the parameters from literature starts to deviate from the experimental measurements 
above $20 \mu \mathrm{m}$ in the $\mathrm{y}$-axis, which indicates that there is room for optimization for model parameters.

To search for optimized parameters, a python script was written to perform an automated Abaqus parametric study. We have conducted 1900 FE simulations where a large number of values in a predetermined range for the strain rate-related parameters to be calibrated ( $\mathrm{C}$ for the JC model, $\alpha_{1}$ and $\beta_{1}$ for the ZA model) were implemented. The ranges for these parameters were selected based on the originally proposed values from literature reported in Table 1 such that they contain the default parameters with at least one order of magnitude higher and lower values being swept in the search. For the JC model, 500 evenly spaced C values between 0.005 and 0.25 were used, resulting in a total of 500 simulations. For the ZA model, 40 evenly spaced $\alpha_{1}$ values between 0 and $3.5 \times 10^{-4}$, and 40 evenly spaced $\beta_{1}$ values between $2.5 \times 10^{-5}$ and $3.5 \times 10^{-}$ ${ }^{4}$ were used, resulting in a total of 1600 simulations. As a result, each simulation produces a deformation profile related to parameters to be calibrated, which we can compare with the experimental measurements.

To quantitively establish the level of agreement between each simulation and the experimental results, we define the goodness of fit, G [20]:

$$
G=1-\sqrt{\frac{\sum_{i=1}^{n} \delta_{i}^{2}}{\sum_{i=1}^{n}\left(\delta_{i}-\delta_{a v g}\right)^{2}}},
$$

where $\delta_{i}$ is the length of the line segment resulting from the intersections of radial line $i$ from $(0,0)$ coordinate with the simulated profile and the experimental profile, as 
graphically illustrated in Fig. 5B. A total number of $n$ radial lines is constructed, and $\delta_{\text {avg }}$ is the average length of $n$ line segments. In our case, 100 evenly spaced radial lines were constructed between $2^{\circ}$ and $88^{\circ}$ to cover the curved part of the deformed profiles. Consequently, a perfect agreement, meaning that the deformed particle's shape generated from simulation exactly matches that observed in the experiment, would yield a $\mathrm{G}$ value equal to 1 . Otherwise, a higher $\mathrm{G}$ value corresponds to a better agreement. Therefore, the goodness of fit is a quantitative measure of the level of agreement between the simulated deformation and that observed from experiments. We consider the value(s) for the calibrated parameter(s) generating the highest $\mathrm{G}$ value as the "optimized" value(s).

A

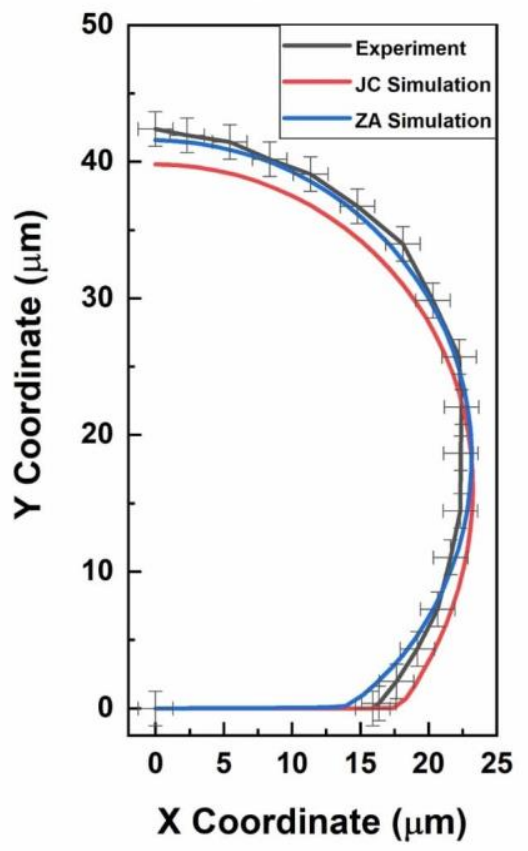

B

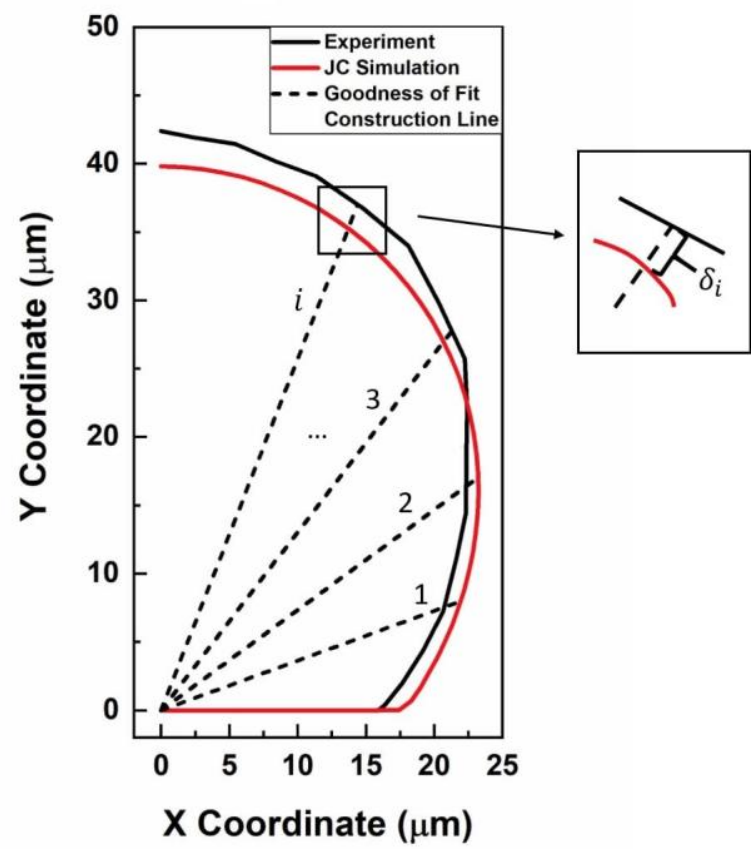

Figure 6: Particle geometries reconstructed for (A) comparison among experimental observations, simulated results from the JC model with material parameters proposed 
in the literature and simulated results from the ZA model with material parameters proposed in the literature. (B) Graphical representation of the line segments used for calculation of the "goodness of fit."

Figure 7 shows the goodness of fit changes as a function of the strain rates sensitivity parameter, $\mathrm{C}$, in the $\mathrm{JC}$ constitutive equation. While the literature value, i.e., $\mathrm{C}=$ 0.0194 , generates a goodness of fit, $G=0.7794$, the highest goodness of fit, $G=$ 0.9321, was obtained at $\mathrm{C}=0.0894$, which we consider to be the optimized $\mathrm{C}$ for the JC model. In this case, we were able to obtain about $20 \%$ improvement in the goodness of fit with an increase in $\mathrm{C}$ by a factor of 4.6. We attribute this significant increase in the strain rate sensitivity parameter to the fact that this parameter is usually originally calibrated through conducting Kolsky bar experiments at for the different strain rate regime, i.e., $10^{2}-10^{4} \mathrm{~s}^{-1}[13-15]$. The strain rate in our impact experiment is significantly different (mainly beyond $10^{6} \mathrm{~s}^{-1}[25]$ ), where titanium, like many other metallic materials, is expected to show an increased strain rate sensitivity compared to lower strain rate regimes $[14,15,21,22]$. At the strain rates of our impact experiment, a considerably higher flow stress is needed to deform material than those predicted by a simple extrapolation of the experimental measurements at lower strain rates induced in the Kolsky bar experiments. This is also apparent in Fig. 7, where we observe less deformation in the experimental geometry while simulated geometry from the JC model with initial $\mathrm{C}$ value produced more flattening in the particle. Our results show that calibration of the strain rate sensitivity term at lower strain rates provided by Kolsky cannot accurately represent the deformation at higher strain rates and that in 
the case of titanium a $4 \mathrm{X}$ increase in the strain rate sensitivity parameter is needed to account for deformation at strain rates beyond $10^{6} \mathrm{~s}^{-1}$.

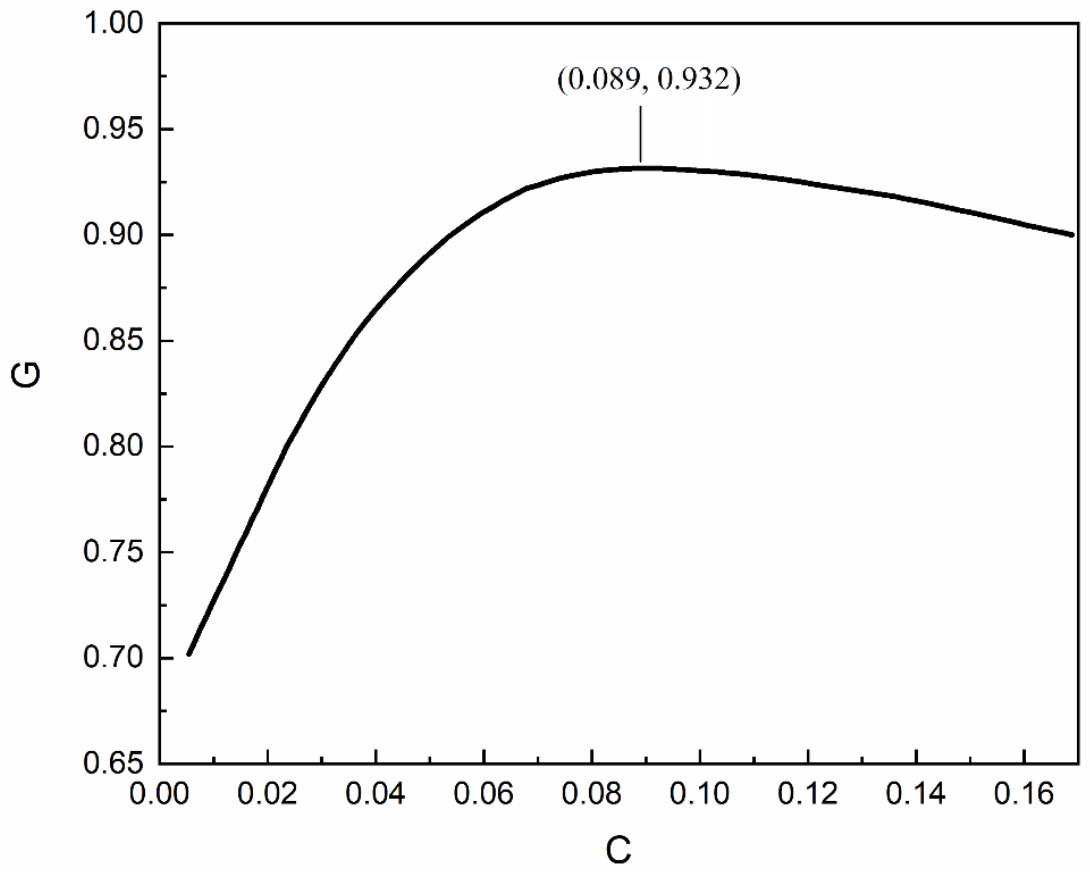

Figure 7: A total of 500 "G values" plotted as a function of C from the JC model.

Out of 1600 total simulations performed for the ZA model, 1492 were completely converged and 108 resulted in a large distortion of the elements. We attribute the latter to extremely out of range values used for $\alpha_{1}$ and $\beta_{1}$ in our optimization search and excluded them from subsequent analysis. Figure 8 shows a contour map of the goodness of fit as a function of $\alpha_{1}$ and $\beta_{1}$ from the 1492 successful simulations. The goodness of fit generated by the original set of $\alpha_{1}$ and $\beta_{1}$ taken from Xue et al. [24] is calculated to be $G=0.9128$ while the highest value for the goodness of fit, $G=0.9212$ was achieved at $\alpha_{1}=0$ and $\beta_{1}=1.25 \times 10^{-4}$. The small increase in the goodness of fit $(\sim 1 \%)$ indicates the ability of the ZA model in describing the flow behavior of CP Ti 
at high strain rates. It is noteworthy that Xue et al. [24] calibrated the ZA model with experimental strain rates in the range of $10^{3}-10^{4} \mathrm{~s}^{-1}$. Yet, an extrapolation to strain rates beyond $10^{6} \mathrm{~s}^{-1}$ proves valid which is rooted in the physical origin of the ZA equation unlike the empirical nature of the JC equation.

Unlike the optimization for the JC model where an increase in C was observed, a decrease in $\alpha_{1}$ to 0 , along with an increase in $\beta_{1}$, was obtained for the ZA model to arrive at the optimized value. Bearing in mind that the functional form of the ZA equation for hep metals is a superposition of those for BCC and FCC metals [24], and that the strain rate sensitivity effect contributed by FCC type deformation response is effectively 0 in the optimized condition, our results suggest that the deformation response of the $\mathrm{CP}$ Ti under ultra-high strain rate is solely determined by that of BCCtype contribution, which agrees with Holt et al. [38]. As BCC metals exhibit higher strain rate sensitivity than FCC metals[35], the increase in strain rate sensitivity of titanium plastically deforming at this high strain rate leads to a strain rate dependence dominated by the BCC contribution. As a result, by setting $\alpha_{1}$ equal to zero and increasing $\beta_{1}$, we were able to calibrate the ZA model that yielded better simulation results for hcp metals undergoing an ultra-high deformation rate. 


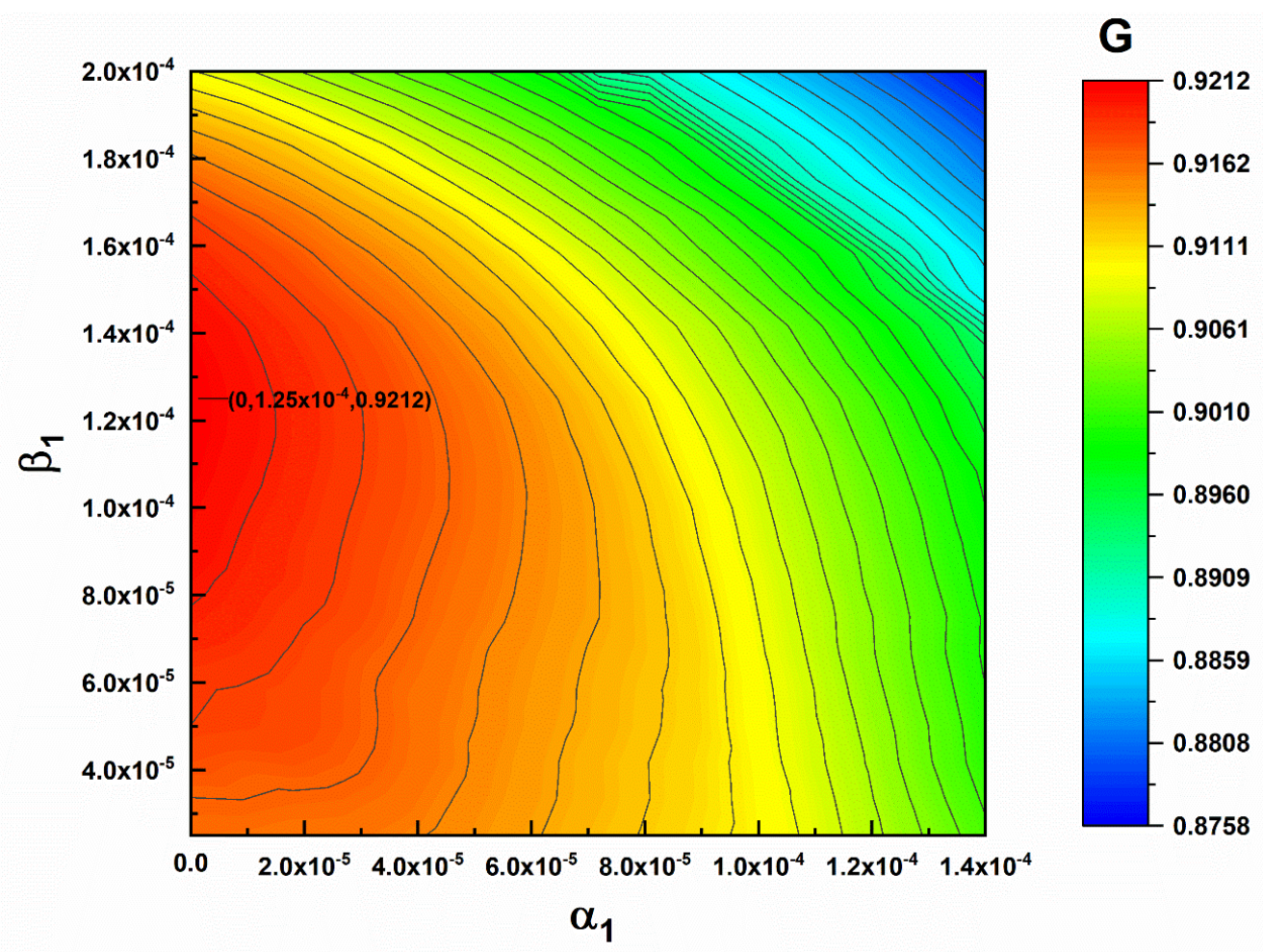

Figure 8: Map of the goodness of fit as a function of the ZA model strain rate sensitivity parameters, $\alpha_{1}$ and $\beta_{1}$.

To calculate the strain rate regime experienced during our impact experiments, we adopt the approach proposed by Burley et al. [20], who present a formulation to calculate the plastic work done at a range of strain rates. From the FE simulation, one can obtain the stress, incremental strain, and strain rate for each volume element at every time interval, from which the incremental plastic work done in the $j_{t h}$ volume element at $k_{t h}$ time increment can be calculated according to:

$$
\Delta \mathrm{W}_{j, k}=\sigma_{j, k} \Delta \varepsilon_{j, k} v_{j}
$$

where $\sigma$ is the stress, $\Delta \varepsilon$ is the incremental strain, and $v$ is the elemental volume. As a result, one can find the total plastic work done by summing over the indices $j$ and $k$. If 
$\Delta \mathrm{W}_{j, k}$ is categorized according to its corresponding strain rate into different strain rate range, one can show the strain rate ranges within which a specific amount of plastic work is done.

Figure 9 shows the fractions of plastic work done during the impact at corresponding strain rates. It can be seen that the range of strain rates under which plastic deformation occurred is between $10^{6}$ and $10^{10} \mathrm{~s}^{-1}$. In fact, the majority of plastic deformation (almost 70\%) occurred with a strain rate between $10^{8}$ and $10^{9} \mathrm{~s}^{-1}$, and about $8.5 \%$ deformation experienced a strain rate that exceeds $10^{9} \mathrm{~s}^{-1}$. This indeed confirms that our experimental approach is capable of generating deformation rates that are well above those produced by traditional test methods, making the investigation of material response and calibration of material models at ultra-high strain rates feasible. Also, it establishes that, in our experiment, the plastic deformation strain rate is beyond $10^{6} \mathrm{~s}^{-1}$ with the majority of the deformation occurring beyond $10^{8} \mathrm{~s}^{-1}$. 


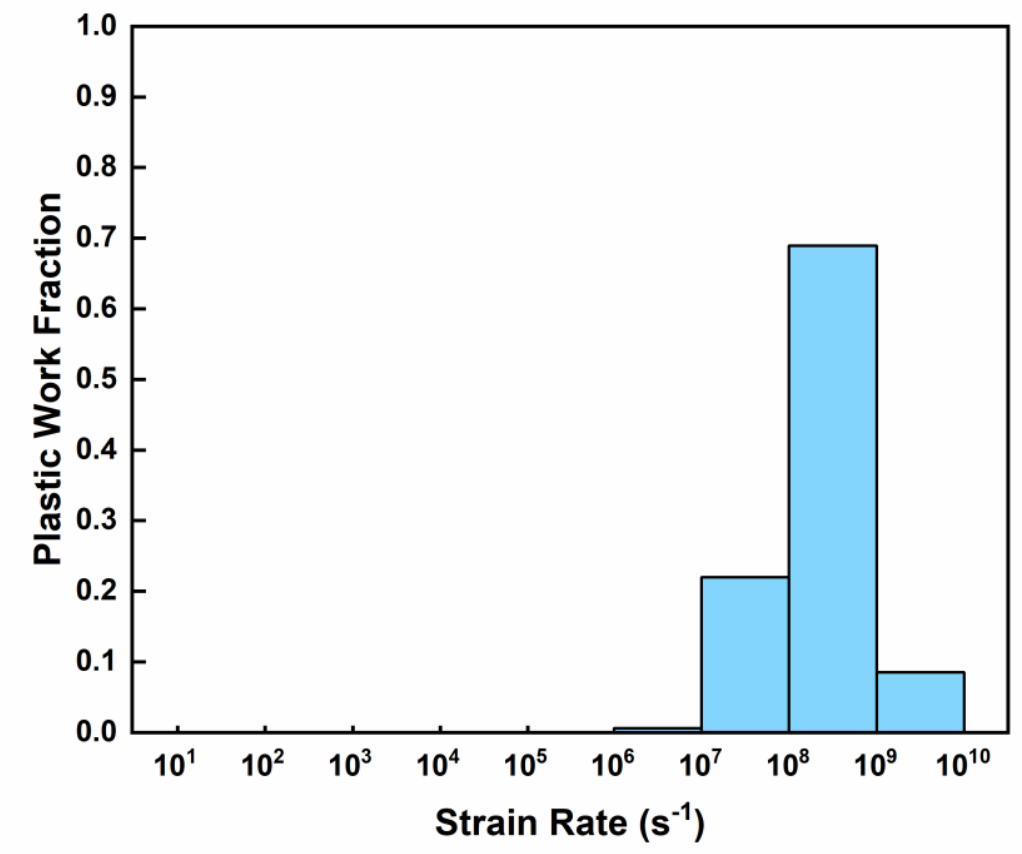

Figure 9: The percentage of plastic work done at different strain rate ranges.

The deformed particle geometries from simulations using both the JC model and the ZA model with the optimized material parameters are plotted in Fig. 10 and compared with the experimental measurements. Figure 10 clearly shows that all three profiles agree with each other very well. The deformed particles from simulations of different material models now are almost identical, with a much better improvement for the JC model. The optimized geometries agree with the numerical results in terms of the level of improvement we obtained after optimization; while there is observable change in particle profile for the JC model to better match the experiment geometry, little change in the particle's shape for the ZA model can be seen as a result of the good agreement that already existed with literature values for model parameters. 


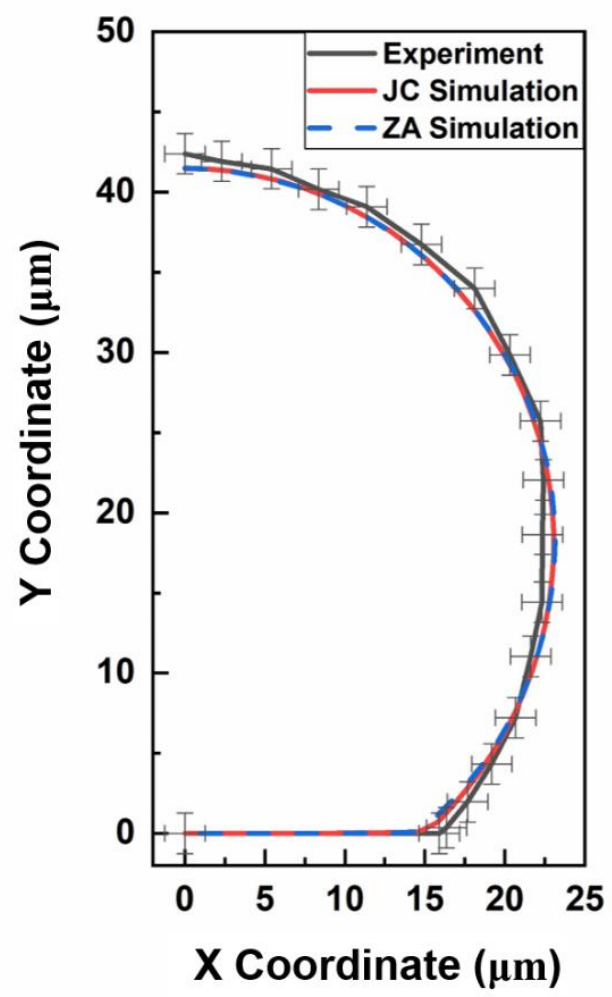

Figure 10: Geometry comparison among experimental observations, simulated results from the JC and ZA models with optimized material parameters.

To further verify the optimization approach adopted in this study, we also performed analysis for both the JC and the ZA models on a wider range of impact velocities, from 144 to $428 \mathrm{~m} / \mathrm{s}$. We measured both impact and rebound velocities $\left(\mathrm{V}_{\mathrm{i}}\right.$ and $\left.\mathrm{V}_{\mathrm{r}}\right)$ form real-time observation and plotted the coefficient of restitution, defined as e $=$ $\mathrm{V}_{\mathrm{r}} / \mathrm{V}_{\mathrm{i}}$, as a function of impact velocity in Fig. 11, in which the data points are scaled to the impacting particle's size of the corresponding experiment, ranging from $22.4 \mu \mathrm{m}$ to $67.2 \mu \mathrm{m}$. We have conducted corresponding finite element simulations with the exact particle size and impact velocity for each impact experiment. The coefficients of restitution from the simulations with the $\mathrm{JC}$ and $\mathrm{ZA}$ constitutive equations and the 
original and optimized strain rate parameters are also superimposed to the plots of Fig. 10. In line with experimental measurements, both models predict that the coefficient of restitution decreases as the impact velocity increases. We note that the JC model with the original strain rate parameter tends to underestimate the coefficient of restitution while the ZA model with the original strain rate parameter tends to slightly overestimate the coefficient of restitution. However, the optimized strain rate parameters were able to improve the prediction with the modeling data points shifting toward the experimental measurements. We observe that the optimization on the JC constitutive equation generated more improvement in terms of the agreement with the experimental measurements compared to the ZA constitutive equation, while the original and the optimized parameters for the ZA model both yielded relatively good results. Therefore, the analysis of the coefficient of restitution not only is in line with the geometrical comparisons presented previously but also confirms that the optimized strain rate parameters can be used to model impact-induced deformation at a wider range of impact velocities. 

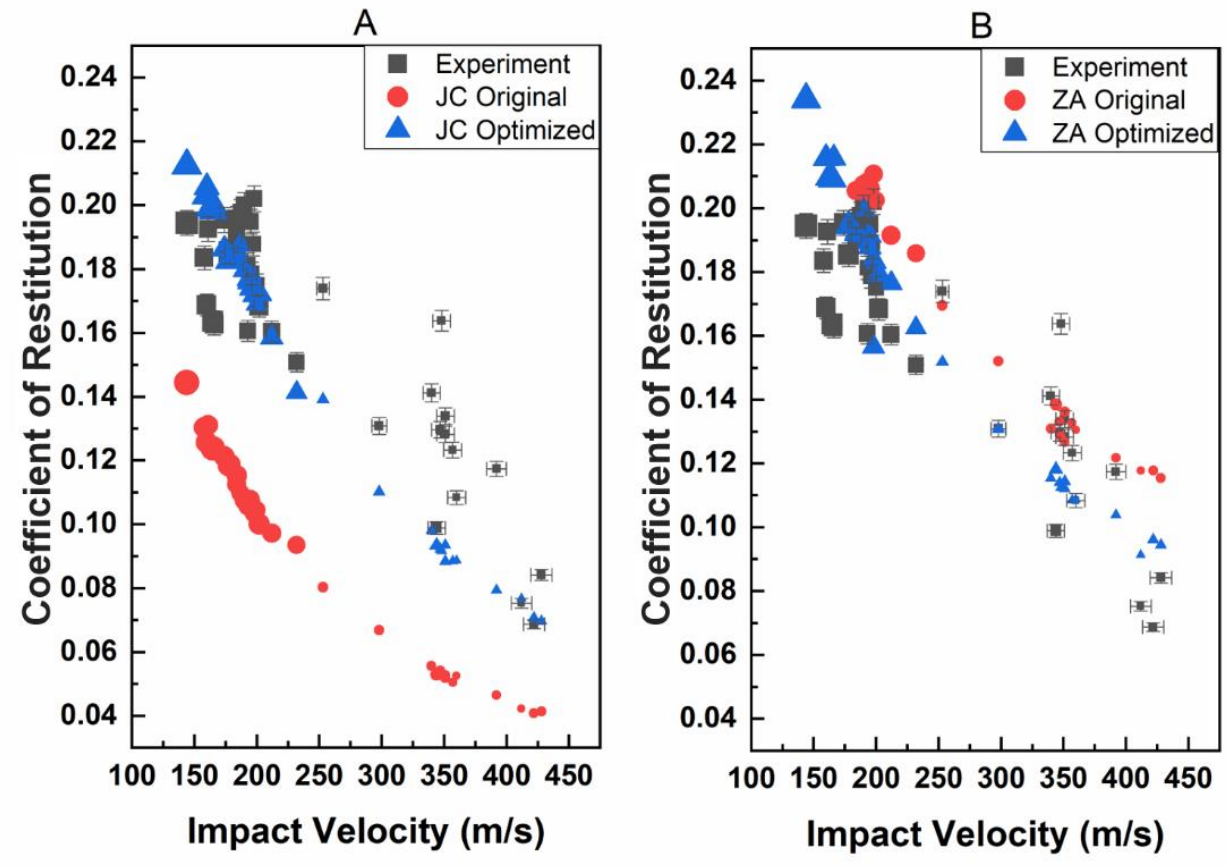

Figure 11: Coefficient of restitution as a function of impact velocity plotted for experimental data, the original and optimized JC model (left) and the original and optimized ZA model (right).

\subsection{Analytical Framework}

In the previous section, we have used finite element simulations for calibration of the strain rate sensitivity parameters. In what follows we propose a simpler framework to approximate the strain rate sensitivity parameter to the first order if the real-time observations of the impact-induced deformation of a spherical object are in hand. Wu et al. [41] proposed a functional form that relates the coefficient of restitution, defined as the ratio of rebound velocity to impact velocity $\left(V_{r} / V_{i}\right)$, to the impact velocity for the impacts of elastic-perfectly plastic spherical particle upon rigid targets: 


$$
\mathrm{e}=0.62\left(\frac{V_{i} / V_{y}}{E^{*} / Y}\right)^{-\frac{1}{2}}
$$

where $V_{y}$ is the critical impact velocity at which the impacting particle starts to yield, $Y$ is the dynamic yield strength and $E^{*}$ is the combined Young's Modulus defined as:

$$
\frac{1}{E^{*}}=\sum_{i=1}^{2} \frac{1-v_{i}^{2}}{E_{i}}
$$

where $v_{i}$ and $E_{i}$ are Poisson's ratio and Young's Modulus of the particle and the substrate, respectively. Also, Johnson [42] has given a relationship between $V_{y}$ and $Y$ :

$$
V_{y}=5.099\left(\frac{Y^{5}}{E^{* 4} \rho}\right)^{\frac{1}{2}}
$$

where $\rho$ is the density of the impacting particle. Combining Equations 6 and 8, we obtain the following equation:

$$
\mathrm{Y}=\left(\frac{V_{i} E^{*} \rho^{\frac{1}{2}}}{5.099\left(\frac{e}{0.62}\right)^{-2}}\right)^{\frac{2}{3}}
$$

which relates the dynamic yield strength of the impacting particle to the impact velocity, the coefficient of restitution, and the properties of the particle and substrate materials. We propose to equate the dynamic yield strength obtained from this calculation with the constitutive equation of interest. With simplifying assumptions on the effective strain rate, effective plastic strain and effective temperature rise and 
simplifications, we can then estimate a model parameter of choice (strain rate sensitivity in the present case) for the considered constitutive equation.

In what follows we apply this framework to estimate the strain rate sensitivity parameter in the JC constitutive equation. Since Eq. 8 is valid only for elastic-perfectly plastic materials, we simplify the JC equation by eliminating the strain hardening term:

$$
Y\left(\dot{\varepsilon_{p}}, T\right)=[A]\left[1+\operatorname{Cln}\left(\dot{\varepsilon_{p}}{ }^{*}\right)\right]\left[1-\left(T^{*}\right)^{m}\right] .
$$

We estimate the effective strain rate for each impact as being the ratio of the impact velocity and particle size (Eq. 11). Furthermore, we calculate the plastic work as the difference between the energy of impacting and rebounding particles and assume that $90 \%$ of the plastic work is dissipated as heat. Therefore, we use Eq. 12 to estimate the effective temperature rise as a result of the impact.

$$
\begin{gathered}
{\dot{\varepsilon_{p}}}^{*}=V_{i} / d \\
T=T_{0}+\frac{0.9\left[\frac{1}{2}\left(V_{i}^{2}-V_{r}^{2}\right)\right]}{c} .
\end{gathered}
$$

Equations 11 and 12 complete the set of equations that allows calculations of strain rate sensitivity parameter, $\mathrm{C}$, for each experiment. These calculations for a total of 34 impact experiments in the present work lead to an average value of $\mathrm{C}=0.0618$ for the strain rate parameter in the JC equation with a standard deviation of 0.0185 . The $\mathrm{C}$ value calibrated using this approximate but handy framework is relatively close to the 
optimized value (0.0895) and corresponds to a $G$ value that is over 0.9 as shown in Fig. 6. Therefore, the framework proposed here is able to estimate a constitutive parameter to first-order without simulation effort while producing a reasonable result. However, we highlight that the approach is only valid if the constitutive equation can be reduced to a form that is independent of plastic strain with relatively little change to the model as a whole such as the JC model.

\section{Conclusion}

With a decrease in projectile length scale to the micron scale, we have induced ultrahigh strain rates in the range of $10^{6}-10^{10} \mathrm{~s}^{-1}$ in spherical microparticles of titanium impacting a rigid substrate. We have recorded impact-induced deformation of the microparticles in real time and simulated the deformation using a finite element approach and two constitutive equations of Johnson-Cook and Zerilli-Armstrong. By comparing the deformed geometries from experimental data and simulated results we have evaluated the capability of the two constitutive equation-originally calibrated at $10^{3}-10^{4} \mathrm{~s}^{-1}$ - to describe deformation at ultrahigh strain rates. The Zerilli-Armstrong

model was found to have a better performance than the Johnson-Cook model at higher strain rates; we found that an extrapolation of the Johnson-Cook strain rate sensitivity to higher strain rate leads to a significant deviation from the experimental geometry whereas the deviation for the Zerilli-Armstrong was negligible. We developed an optimization scheme that searched for the optimum strain-rate parameters that could best predict the experimental geometry. With the improved strain rate sensitivities, we achieved about 20\% improvement in the Johnson-Cook. We have also found the BCC- 
type contribution to the strain rate sensitivity of pure titanium dominates the BCC-type contribution in the Zerilli-Armstrong form proposed for HCP metals. The improved strain rate parameters for both Johnson-Cook and Zerilli-Armstrong were found to successfully predict the coefficient of restitution of titanium microparticles impacting rigid substrate in the velocity range of $144-428 \mathrm{~m} / \mathrm{s}$. We have finally developed a simplified analytical framework that to the first order can calibrate the strain rate sensitivity from the experimental coefficients of restitution of FE simulations. 


\section{Reference}

[1] Field, J. E., Walley, S. M., Proud, W. G., Goldrein, H. T., and Siviour, C. R., 2004, Review of Experimental Techniques for High Rate Deformation and Shock Studies.

[2] Camacho, G. T., and Ortiz, M., 1997, “Adaptive Lagrangian Modelling of Ballistic Penetration of Metallic Targets," Comput. Methods Appl. Mech. Eng., 142(3-4), pp. 269-301.

[3] Schmidt, T., Gärtner, F., Assadi, H., and Kreye, H., 2006, "Development of a Generalized Parameter Window for Cold Spray Deposition," Acta Mater., 54(3), pp. 729-742.

[4] Mellal, M. A., and Williams, E. J., 2016, "Parameter Optimization of Advanced Machining Processes Using Cuckoo Optimization Algorithm and Hoopoe Heuristic,” J. Intell. Manuf., 27(5), pp. 927-942.

[5] Miao, H. Y., Larose, S., Perron, C., and Lévesque, M., 2009, "On the Potential Applications of a 3D Random Finite Element Model for the Simulation of Shot Peening," Adv. Eng. Softw., 40(10), pp. 1023-1038.

[6] Lim, C. T., Shim, V. P. W., and Ng, Y. H., 2003, "Finite-Element Modeling of the Ballistic Impact of Fabric Armor," Int. J. Impact Eng., 28(1), pp. 13-31.

[7] Driscoll, D. E., ed., 1970, Impact Testing of Metals, ASTM International, West Conshohocken, PA.

[8] Taylor, G., 1948, "The Use of Flat-Ended Projectiles for Determining Dynamic Yield Stress I. Theoretical Considerations,” Proc. R. Soc. London. Ser. A. Math. Phys. Sci., 194(1038), pp. 289-299.

[9] Hopkinson, B., 1914, "A Method of Measuring the Pressure Produced in the Detonation of High Explosives or by the Impact of Bullets," Philos. Trans. R. Soc. A Math. Phys. Eng. Sci.

[10] Gray III, G. T., 2000, "Shock Wave Testing of Ductile Materials," ASM Handbook: Mechanical Testing and Evaluation, Vol. 8., H. Kuhn, and D. Medlin, eds., Materials Park, OH: ASM International, pp. 530-538. 
[11] Murugesan, M., and Jung, D. W., 2019, “Johnson Cook Material and Failure Model Parameters Estimation of AISI-1045 Medium Carbon Steel for Metal Forming Applications," Materials (Basel)., 12(4).

[12] Sheikh-Ahmad, J. Y., and Bailey, J. A., 1995, "A Constitutive Model for Commercially Pure Titanium," J. Eng. Mater. Technol. Trans. ASME, 117(2), pp. 139-144.

[13] Simon, P., Demarty, Y., Rusinek, A., and Voyiadjis, G. Z., 2018, "Material Behavior Description for a Large Range of Strain Rates from Low to High Temperatures: Application to High Strength Steel," Metals (Basel)., 8(10).

[14] Chichili, D. R., Ramesh, K. T., and Hemker, K. J., 1998, “The High-Strain-Rate Response of Alpha-Titanium: Experiments, Deformation Mechanisms and Modeling," Acta Mater.

[15] Mishra, A., Martin, M., Thadhani, N. N., Kad, B. K., Kenik, E. A., and Meyers, M. A., 2008, "High-Strain-Rate Response of Ultra-Fine-Grained Copper," Acta Mater.

[16] Gangireddy, S., 2018, “A Modified Johnson-Cook Model for Cp-Ti to Incorporate the Effects of Dynamic Strain Aging and Phase Transformation," Int. J. Metall. Met. Phys., 3(2), pp. 1-11.

[17] Meyer, H. W., 2006, "A Modified Zerilli-Armstrong Constitutive Model Describing the Strength and Localizing Behavior of Ti-6Al-4V," Mater. Res., pp. 1-29.

[18] Hernandez, C., Maranon, A., Ashcroft, I. A., and Casas-Rodriguez, J. P., 2013, "A Computational Determination of the Cowper-Symonds Parameters from a Single Taylor Test," Appl. Math. Model., 37(7), pp. 4698-4708.

[19] Dean, J., and Clyne, T. W., 2017, "Extraction of Plasticity Parameters from a Single Test Using a Spherical Indenter and FEM Modelling," Mech. Mater., 105, pp. 112-122.

[20] Burley, M., Campbell, J. E., Dean, J., and Clyne, T. W., 2018, "Johnson-Cook Parameter Evaluation from Ballistic Impact Data via Iterative FEM Modelling," Int. J. Impact Eng., 112(September 2017), pp. 180-192. 
[21] Andrews, E. W., Giannakopoulos, A. E., Plisson, E., and Suresh, S., 2001, "Analysis of the Impact of a Sharp Indenter," Int. J. Solids Struct., 39(2), pp. 281-295.

[22] Lu, J., Suresh, S., and Ravichandran, G., 2003, "Dynamic Indentation for Determining the Strain Rate Sensitivity of Metals," J. Mech. Phys. Solids, 51(11-12), pp. 1923-1938.

[23] Trelewicz, J. R., and Schuh, C. A., 2008, "The Hall-Petch Breakdown at High Strain Rates: Optimizing Nanocrystalline Grain Size for Impact Applications," Appl. Phys. Lett., 93(17), pp. 10-13.

[24] Xue, Q., Meyers, M. A., and Nesterenko, V. F., 2002, "Self-Organization of Shear Bands in Titanium and Ti-6Al-4V Alloy," Acta Mater., 50(3), pp. 575596.

[25] Hassani-Gangaraj, M., Veysset, D., Nelson, K. A., and Schuh, C. A., 2018, "InSitu Observations of Single Micro-Particle Impact Bonding," Scr. Mater., 145, pp. 9-13.

[26] Xie, W., Alizadeh-Dehkharghani, A., Chen, Q., Champagne, V. K., Wang, X., Nardi, A. T., Kooi, S., Müftü, S., and Lee, J. H., 2017, "Dynamics and Extreme Plasticity of Metallic Microparticles in Supersonic Collisions /639/166/988 /639/301/1023/1026 /639/301/930/12 /128 Article," Sci. Rep., 7(1), pp. 1-9.

[27] Veysset, D., Hsieh, A. J., Kooi, S., Maznev, A. A., Masser, K. A., and Nelson, K. A., 2016, "Dynamics of Supersonic Microparticle Impact on Elastomers Revealed by Real-Time Multi-Frame Imaging," Sci. Rep., 6(January), pp. 1-7.

[28] Hassani-Gangaraj, M., Veysset, D., Nelson, K. A., and Schuh, C. A., 2017, "Melting Can Hinder Impact-Induced Adhesion," Phys. Rev. Lett., 119, p. 175701 .

[29] Hassani-Gangaraj, M., Veysset, D., Nelson, K. A., and Schuh, C. A., 2016, "Supersonic Impact of Metallic Micro-Particles," arXiv:1612.08081.

[30] Veysset, D., Hsieh, A. J., Kooi, S. E., and Nelson, K. A., 2017, "Molecular Influence in High-Strain-Rate Microparticle Impact Response of Poly(Urethane Urea) Elastomers," Polymer (Guildf)., 123, pp. 30-38. 
[31] Abaqus(2018), “Abaqus User Subroutines Reference Guide.”

[32] Yıldırım, B., and Müftü, S., 2012, "International Journal of Solids and Structures Simulation and Analysis of the Impact of Micron-Scale Particles onto a Rough Surface," 49, pp. 1375-1386.

[33] Johnson G.R., C. W. H., 1983, “A Constitutive Model and Data for Metals.Pdf," Proc. seventh Int. Symp., pp. 541-547.

[34] Zerilli, F. J., and Armstrong, R. W., 1987, "Dislocation-Mechanics-Based Constitutive Relations for Material Dynamics Calculations,” J. Appl. Phys., 61(5), pp. 1816-1825.

[35] Meyers, M., 1994, "Dynamic Behavior of Materials," Dynamic Behavior of Materials, John Wiley \& Sons, Inc.

[36] Vöhringer, O., 1989, "Deformation Behavior of Metallic Materials," International Summer School on Dynamic Behavior of Materials, Nantes.

[37] HOLMQUIST, T. J., and JOHNSON, G. R., 1991, "Determination of Constants and Comparison of Results for Various Constitutive Models," Le J. Phys. IV, 01(C3), pp. C3-853-C3-860.

[38] Holt, W. H., Mock, W., Zerilli, F. J., and Clark, J. B., 1994, "Experimental and Computational Study of the Impact Deformation of Titanium Taylor Cylinder Specimens," Mech. Mater., 17(2-3), pp. 195-201.

[39] Zerilli, F. J., and Armstrong, R. W., 1995, “.,” Shock Compression of Condensed Matter, S.C. Schmidt, and W.C. Tao, eds., AIP Conference Proceedings (no. 370), Seattle (WA), pp. 31-35.

[40] Bae, G., Xiong, Y., Kumar, S., Kang, K., and Lee, C., 2008, "General Aspects of Interface Bonding in Kinetic Sprayed Coatings," Acta Mater., 56(17), pp. 4858-4868.

[41] Wu, C., Li, L., and Thornton, C., 2003, "Rebound Behaviour of Spheres for Plastic Impacts,” 28(January), pp. 929-946. 
[42] Johnson, K. L., 1987, "Dynamic Effects and Impact," Contact Mechanics, Cambridge University Press, pp. 340-373. 